\title{
"A Latino Advantage in Oral Health-Related Quality of Life is Modified by Nativity Status"
}

\author{
Anne E Sanders \\ The University of North Carolina at Chapel Hill, Chapel Hill, NC UNITED STATES \\ Anne E Sanders: anne_sanders@dentistry.unc.edu
}

\section{Abstract}

Explanations for the social gradient in health status are informed by the rare exceptions. This crosssectional observational study examined one such exception, the "Latino paradox" by investigating the presence of a Latino advantage in oral health-related quality of life and the effect of nativity status on this relationship. A nationally representative sample of adults $(n=4208)$ completed the National Health and Nutrition Examination Survey (NHANES) 2003-2004. The impact of oral disorders on oral health-related quality of life was evaluated using the NHANES Oral Health Impact Profile.

Exposures of interest were race, ethnicity and nativity status. Covariates included sociodemographic characteristics, smoking status, self-rated health, access to dental care and number of teeth.

Unconditional logistic regression models estimated odds of impaired oral health-related quality of life for racial/ethnic and nativity groups compared to the Non-Latino white population. Overall prevalence of impaired oral health-related quality of life was $15.1 \%$. A protective effect of Latino ethnicity was modified by nativity status, such that Latino immigrants experienced substantially better outcomes than non-Latino whites. However the effect was limited to first-generation Latinos. U.S. born Latinos did not share the oral health-related quality of life advantage of their foreign-born counterparts. This advantage was not attributable to the healthy migrant phenomenon since immigrants of non-Latino origin did not differ from Non-Latino whites. The excess risk among NonHispanic Blacks was rendered non-significant after adjustment for socioeconomic position. A protective effect conferred by Latino nativity is unexpected given relatively disadvantaged socioeconomic position of this group, their language barrier and restrictions to needed dental care. As the Latino advantage in oral health-related quality of life is not explained by healthy immigrant selection, cultural explanations seem more likely than explanations based on characteristics of individuals.

\section{Keywords}

USA; Acculturation; Hispanic; Disparities; Epidemiology; Social Class; Oral Health; nativity; Latino paradox; ethnicity

Publisher's Disclaimer: This is a PDF file of an unedited manuscript that has been accepted for publication. As a service to our customers we are providing this early version of the manuscript. The manuscript will undergo copyediting, typesetting, and review of the resulting proof before it is published in its final citable form. Please note that during the production process errors may be discovered which could affect the content, and all legal disclaimers that apply to the journal pertain. 


\section{INTRODUCTION}

One of the most robust epidemiologic norms is that health status is inversely related to social and economic standing. This socioeconomic gradient applies to almost all dimensions of health including oral health (Borrell \& Crawford, 2008; Sabbah, Tsakos, Chandola, Sheiham, \& Watt, 2007). Recently, efforts have shifted from describing the gradient toward conceptual framework to guide explanations for its persistence and formulate strategies to reduce the health disparities it produces (Patrick, Lee, Nucci, Grembowski, Jolles, \& Milgrom, 2006).

A well-documented exception to this gradient is the so-called Latino paradox; that despite lower educational attainment and higher rates of poverty, Latinos on average enjoy an equal or better health status than the non-Latino white population in the United States (U.S.). Examination of exceptions such as these may provide clues about causal mechanisms that underlie the social gradient in health. To date strongest evidence for the Latino health advantage is for infant mortality rate and age-adjusted all-cause mortality rate; two highly sensitive markers of economic development and health in populations (Abraido-Lanza, Dohrenwend, Ng-Mak, \& Turner, 1999; Hummer, Powers, Pullum, Gossman, \& Frisbie, 2007; Liao, Cooper, Cao, Durazo-Arvizu, Kaufman, Luke et al., 1998; Markides \& Eschbach, 2005; Sorlie, Backlund, Johnson, \& Rogot, 1993; Thomas, Eberly, Neaton, \& Smith, 2005; Turra \& Elo, 2008; Turra \& Goldman, 2007). An early explanation, now discounted, dismissed the Latino mortality advantage as a statistical artefact arising from misclassification of ethnicity from census data and deaths from vital statistics (Arias, Schauman, Eschbach, Sorlie, \& Backlund, 2008). Two contemporary explanations involve migratory selection effects. The salmon bias hypothesis contends that return migration to country of birth is selective of poor health. Foreign deaths are not recorded in U.S. mortality registries (except those in Puerto Rico), artificially lowering the Latino mortality rate. The healthy migrant effect observes that people who succeed in securing residence in the U.S. are a non-random sample from their country of origin, positively selective for health, education and other characteristics that promote adaptation to the host nation. Despite plausibility of selection effects, the empirical support is weak. For instance in a cohort comparison of pre- and post- migration health status, the Mexican Family Life Survey found little evidence in a nationally representative sample of young adults, that migrants to the U.S. were any healthier or better educated than those who did not migrate (Rubalcava, Teruel, Thomas, \& Goldman, 2008). Other studies conclude that salmon bias and the healthy migrant effect contribute to the Latino health advantage, but fail to fully account for it (Abraido-Lanza et al., 1999; Franzini, Ribble, \& Keddie, 2001; Turra \& Elo, 2008).

Latinos are also advantaged in perinatal outcomes such as preterm birth and low birthweight (Hessol \& Fuentes-Afflick, 2000), chronic disorders such as asthma (Moorman, Rudd, Johnson, King, Minor, Bailey et al., 2007; Subramanian, Jun, Kawachi, \& Wright, 2009), and psychiatric disorders (Alderete, Vega, Kolody, \& Aguilar-Gaxiola, 2000; Alegria, Canino, Shrout, Woo, Duan, Vila et al., 2008). However certain disorders, notably diabetes and parasitic diseases, show higher rates among Latinos than among the white non-Latino population.

Most studies of the Latino health advantage have focused on mortality and morbidity. One aspect that remains unexamined is whether the Latino health advantage extends to appraisals of the impact of health on aspects of daily living. To address this gap, the objective of this study was to compare how Latinos appraise the impact of their dental conditions on oral healthrelated quality of life relative to other racial/ethnic groups in the U.S. If a Latino advantage was found in oral health-related quality of life, this study aimed to determine whether the effect was modified by nativity status. 


\section{METHODS}

\section{Study and sampling designs and data collection}

The National Health and Nutrition Examination Survey (NHANES) is a continuous crosssectional survey conducted by the National Center for Health Statistics (NCHS). NCHS uses a complex multistage sampling design to produce a nationally representative sample of the civilian non-institutionalized population of the U.S. aged 2 months or older. NHANES 20032004 survey were used in this analysis as these were the first to include a nationwide multiitem assessment of oral health-related quality of life. The NHANES protocol includes a home interview administered by a trained interviewer in English or Spanish, followed by a medical examination with a dental component. The overall response rate to the medical examination was $76 \%(n=9,643)$ and 5,885 of these participants were aged 16 years or older.

\section{Inclusion and exclusion criteria}

Inclusion was restricted to dentate participants aged 18 years or older who completed the home interview and whose teeth were counted $(n=4,377)$. Since the focus was a comparison of Latinos with major racial groups, persons identifying as multiracial, belonging to minor racial groups, or with missing racial/ethnicity data were excluded from analysis $(\mathrm{n}=169)$. Thus this analysis used data for 4,208 NHANES participants.

\section{Main exposure variable}

"Hispanic" in the NHANES protocol refers to Spanish-speaking background. Consequently this term is used in reference to NHANES data; otherwise "Latino" is used, referring to Latin American derivation. The NHANES-derived race/ethnicity variable combined responses to questions on self-identified race and Hispanic origin status. NHANES classified country of origin as United States, Mexico or Other. Data from these two variables were aggregated to derive a new variable that defined racial/ethnic groups according to nativity status. This yielded five categories: Non-Hispanic White, U.S. born; Non-Hispanic White, immigrant; NonHispanic Black; Hispanic, U.S. born; Hispanic, immigrant. Immigrants refer to foreign-born persons. Since few Non-Hispanic Blacks were immigrants $(n=45)$, this category was not separately examined. Nativity status serves as a crude marker of duration of exposure to the U.S.

\section{Dependent variable}

Oral health-related quality of life was assessed in the home interview using the NHANES Oral Health Impact Profile (NHANES OHIP). This seven-item questionnaire is validated as a brief independent scale, comparable in responsiveness and discrimination properties to the 14-item OHIP (Sanders, Slade, Lim, \& Reisine, 2009). The OHIP (Slade, 1997; Slade \& Spencer, 1994) is the most extensively studied oral health-related quality of life scale now translated into over 20 languages. The Spanish translation was found to be valid and consistent when tested in a Chilean sample (Lopez \& Baelum, 2006). The theoretical basis for the OHIP was adapted for oral health (Locker, 1988) from the WHO International Classification of Impairments, Disabilities and Handicaps (World Health Organization, 1980). The NHANES OHIP retains this theoretical basis. The multidimensional questions evaluate the impact of dental disorders on functional impairment (affected taste; food avoidance), physical discomfort (painful aching), psychological impact (embarrassment, self-consciousness) and social restriction (difficulty doing usual jobs; life less satisfying). Responses are recorded on a fivepoint ordinal scale coded $0=$ never, $1=$ hardly ever, $2=$ occasionally, $3=$ fairly often, $4=$ very often. The specific wording of each OHIP question enhances its construct validity and helps investigators and participants interpret its meaning. The prevalence score was computed as the summary statistic defined as the percentage of people responding with "fairly often" or "very 
often" to any one or more questions, and described in this study as impaired oral health-related quality of life.

\section{Covariates}

All covariates, except tooth retention, were self-reported in the home interview and selected for their known association with oral health-related quality of life. Interview language was used as a proxy for cultural identity and extent of acculturation. The implicit assumption was that Spanish speaking Latinos identify less with American culture than those whose interview language preference was English.

Age was measured in years and educational attainment was collapsed into three groups (less than high school, high school diploma, more than high school). Economic status was assessed with the poverty income ratio computed as the ratio of income (numerator) taken as the midpoint of the household income category to the U.S. poverty threshold (denominator). Tertiles of poverty income ratio variable were examined in bivariate analysis (low, moderate, high income) while the continuous variable was used in multivariable models. As a marker of risk behaviour, smoking status was dichotomized into homes where anyone smokes, versus homes where no-one smokes. Self-rated general health was dichotomized to compare the poorest categories (fair, poor) against a healthier referent (excellent, very good, good). Access to dental care was dichotomized based on the ability to obtain needed dental care during the past 12 months (yes/no). Number of teeth was clinically determined in the medical examination and used as a continuous variable in multivariable analysis.

\section{Statistical analysis}

Data were analyzed in STATA statistical software, version 10.1 SE (Stata Corporation, Texas). To take account of the complex survey design, survey estimation commands were used to correct the standard errors of estimates by the Taylor series linearization method. For the $0.03 \%$ of NHANES-OHIP items with missing values, the mean sample value for that item was substituted.

Differences in prevalence of impaired oral health-related quality of life between racial/ethnicity and nativity groups were tested for significance with chi-squared tests. To test the study aim, unconditional logistic regression was used to estimate the association of race/ethnicity and nativity with oral health-related quality of life. Estimates were expressed as the crude odds ratio (OR) with its 95\% confidence interval (CI) using the Non-Hispanic White, U.S. born population group as the referent category.

In the first of four multivariable models, crude associations of race/ethnicity and nativity status with impaired oral health-related quality of life were adjusted for gender, age and interview language. To adjust for effect of socioeconomic position, Model 2 added education and poverty income ratio to the preceding model. Smoking status added to Model 3 assessed the effect of a recognized behavioral risk predictor of periodontal disease and tooth loss. Model 4 added self-rated health, number of retained teeth and access to needed dental care. Model 4 was then rerun substituting immigrant Hispanics as the referent category to determine whether the oral health-related quality of life estimate for other racial/ethnic category differed significantly from this group. Finally a post-estimation command computed predicted probabilities from the logistic regression model for impaired oral health-related quality of life for each category of racial/ethnic nativity group, adjusted for covariates. 


\section{RESULTS}

Non-Hispanic Whites comprised $75.9 \%$ of the study population while Non-Hispanic Blacks $(11.9 \%)$ and Hispanics $(12.2 \%)$ contributed an equal-sized minority. The majority was born in the U.S. (87.3\%). Of the foreign-born participants (12.7\%), 4.6\% was born in Mexico and $8.1 \%$ was born in other countries.

When disaggregated by nativity status, immigrant Non-Hispanic Whites contributed $4.3 \%$ of the overall study population and immigrant Hispanics contributed $7.3 \%$ of the overall study population (Table 1). Overall prevalence of impaired oral health-related quality of life was $15.1 \%$ (Table1). In general, adults with impaired oral health-related quality of life were more likely to be Non-Hispanic, female, and middle aged; to have a lower educational level and household income; to live in a household where someone smoked; face restricted access to needed dental care; and have poorer health and fewer teeth (Table 1). Among the racial/ethnic groups, only Non-Hispanic Blacks had significantly higher odds of impaired oral health-related quality of life ( $\mathrm{OR}=1.36,95 \% \mathrm{CI}=1.12-1.65)$ than Non-Hispanic Whites (Table 1). A significant protective effect for immigrant Hispanics $(\mathrm{OR}=0.58,95 \% \mathrm{CI}=0.41-0.81)$ was not shared by U.S. born Hispanics (OR= 0.78, 95\% CI $=0.78-1.71)$.

In unadjusted analysis, the considerable heterogeneity between racial/ethnic groups was contrasted by a striking similarity of U.S. born Hispanics to Non-Hispanic Blacks with respect to education, income and access to dental care (Table 2). On these characteristics, these two groups fared uniformly better than immigrant Hispanics and uniformly worse than nonHispanics whites, irrespective of the nativity status of Whites. In fact, U.S. born Hispanics bore closer resemblance to Non-Hispanic Blacks than to immigrant Hispanics. Illustrating marked difference in nativity status of Hispanics, 59\% of home interviews were conducted in Spanish for immigrant Hispanics compared with only $3 \%$ for U.S. born Hispanics. Non-completion of high school was elevated two-fold among immigrant Hispanics (53.0\%) compared with U.S. born Hispanics (27.6\%). U.S. born Hispanics were almost twice as likely to be in the high income tertile (23.7\%) than immigrant Hispanics (13.1\%) and rates of smoking were elevated almost three-fold in U.S born Hispanic households (17.1\%) compared with Hispanic immigrants (6.8\%). Of note, smoking prevalence was elevated four-fold (28.1\%) among NonHispanic Blacks relative to immigrant Hispanics (6.8\%) Two shared characteristics of Hispanic groups was their young age relative to Whites and their greater level of tooth retention. Once tooth retention was statistically adjusted for age, however, no differences persisted in mean number of teeth between groups (not tabulated).

A comparison of the individual NHANES OHIP items across the five racial and ethnic groups showed that compared with US-born Non-Hispanic Whites, a lower proportion of Hispanic immigrants reported six of the seven NHANES OHIP items (Table 3). Most notably, the proportion of Hispanic immigrants who reported food avoidance was 2.3-fold lower than the proportion of US-born Non-Hispanic Whites who reported this problem in the last 12 months.

Statistical adjustment for gender, age and interview language covariates (Table 4, Model 1) accentuated the protective effect on oral health-related quality of life of immigrant Hispanic status relative to Non-Hispanic White, observed in crude analysis. Additional adjustment for education and income (Table 4, Model 2) further accentuated this protective effect and also eliminated the excess risk of impaired oral health-related quality of life observed for NonHispanic Blacks relative to Non-Hispanic Whites. Since smoking prevalence was relatively low among immigrant Hispanics, adjustment for smoking (Table 4, Model 3) had a greater protective effect for Non-Hispanic Whites, thus increasing the relative health advantage for immigrant Hispanics. Finally, adjustment for general health, tooth retention and access to 
dental care (Table 3, Model 4) enhanced the immigrant Hispanic advantage (OR $=0.28,95 \%$ $\mathrm{CI}=0.15-0.55)$.

In substituting the immigrant Hispanic group as the referent, all other groups were more than twice as likely to have impaired oral health-related quality of life. The odds ratio for U.S. born Hispanics was 2.87 (1.66-4.98) and odds of impaired oral health-related quality of life were for the other three racial/ethnic and nativity groups odds were more than three times as likely compared with immigrant Hispanics. After adjustment for covariates, the predicted probabilities (PP) of impaired oral health-related quality of life for U.S. born and immigrant Non-Hispanic Whites and Non-Hispanic Blacks were identical $(\mathrm{PP}=0.14)$ (Figure 1) and were significantly lower for immigrant Hispanics $(\mathrm{PP}=0.04,95 \% \mathrm{CI}=0.20-0.04)$.

\section{DISCUSSION}

\section{Key findings}

Despite a bleak socioeconomic profile and restricted access to needed dental care, immigrant Latinos enjoyed a substantial oral health-related quality of life advantage over the majority white non-Latino population in this study. The disaggregation by nativity status revealed two important findings. Firstly, the advantage for first-generation Latinos was not conveyed to U.S. born Latinos, despite the latter's stronger English-language assimilation, higher socioeconomic standing, greater access to dental care and better self-rated health. Secondly, the advantage was not expressed by non-Latino immigrants. Neither was the oral health-related quality of life advantage observed among African Americans, who share a minority status in the U.S. with Latinos along with a disadvantaged socioeconomic standing. In summary, this study identified a Latino advantage in oral health-related quality of life that was modified by nativity status, limited to first-generation Latinos, and at odds with evidence for risks associated with socioeconomic disadvantage, limited access to health care, linguistic isolation and migration readjustment in immigrant families. Also noteworthy was the finding that excess risk of impaired oral health-related quality of life among African Americans was eliminated after statistical adjustment for education and poverty.

Findings of a Latino advantage in oral health-related quality of life build onto prior observations of a Latino immigrant advantage in mortality outcomes and in prevalence of asthma (Singh \& Hiatt, 2006; Subramanian et al., 2009) over their U.S. born counterparts. Results also build on evidence that prevalence of periodontitis among Mexican Americans was half that of NonHispanic Blacks and not significantly different to that of Non-Hispanic Whites (Borrell \& Crawford, 2008).

\section{Mechanisms and explanations}

The Latino immigrant oral health-related quality of life advantage cannot be attributed to a protective effect of younger age. This and other studies using the OHIP questionnaire show that if an age effect exists at all, it is older adults, not younger ones, who enjoy better oral health-related quality of life (Sanders, Slade, John, Steele, Suominen-Taipale, Lahti et al., 2009a; Steele, Sanders, Slade, Allen, Lahti, Nuttall et al., 2004). Neither is the oral healthrelated quality of life advantage explained by Latinos' lower rate of smoking and higher rate of tooth retention, since adjustment for these factors did not attenuate risk in the other major groups. Furthermore it is unlikely that the advantage is due to subjective reporting bias since the proportion of immigrant Latinos who rated their health unfavourably was double that of the white non-Latino population and almost triple that of non-Latino immigrants. Moreover the healthy immigrant hypothesis does not account for the effect entirely since other foreignborn immigrants did not enjoy the same oral health-related quality of life advantage at Latino immigrants. 
Something else about Latino immigrant status confers a salutary effect. Explaining this effect has public health salience for promoting resilience to poverty. At the macro-level, differences in political social and economic policy between the U.S. and Latin America may contribute to the explanation. Unlike the U.S., many Latin American countries have had a long tradition of comprehensive systems of social protection. The state has had a strong role in the provision of pension plans and sickness benefits (Coburn, 2004; Moreno-Brid, Pardinas Carpizo, \& Ros Bosch, 2009; Segura-Ubiergo, 2007).

Elsewhere there is evidence that political economies may influence oral health-related quality of life. For example, in a cross-national comparison of countries with different welfare state regimes, income inequalities in oral health-related quality of life were steeper in countries offering a flat rate of benefits to only the poor, than in countries offering earnings-related levels benefits to all citizens (Sanders, Slade, John, Steele, Suominen-Taipale, Lahti et al., 2009b). Given this finding, it is reasonable to speculate that this style of welfare state regime may be protective of oral health-related quality of life among Latino immigrants in the short term.

The Latino advantage may also stem from the strong socio-centric values instilled at familial level. The protective effects on health, including oral health-related quality of life, of reciprocity, social ties and attachments are well recognized (Brennan \& Spencer, 2009;

Melchior, Berkman, Niedhammer, Chea, \& Goldberg, 2003). Less well investigated are values such as familismo (collective loyalty to extended family that brings ties, obligations, and interdependence) and respeto (deference) and educación (moral education). One recent study found that U.S. born Latinos report lower familial social support than their immigrant counterparts (Almeida, Molnar, Kawachi, \& Subramanian, 2009). This implies a weakening of familial ties and attachments with time spent in the U.S. Related to this shift in family ties is the transition from extended families to nuclear families that typically accompanies migration to the U.S. This transition represents a move from inter-dependence towards greater individualism. Such changes, while not necessarily risk factors in themselves, are signals of change which may erode the protective effect of socio-centric values on oral health quality of life. Taken together, these factors may contribute to an understanding of how the initial health advantage in Latino immigrants falters in the second and subsequent generations, concomitant with closure of the education and income gap.

Selection effects again offer another explanation. It is possible that the healthiest U.S. born Latinos most rapidly assimilate into the culture and values of American society and cease to self-identify as Latino, thus lowering the average health status of the U.S. born Latino population. Another possibility is that a withdrawal from the protective cultural elements of Latin America induces a dissipation of the oral helth quality of life benefit.

\section{Limitations}

The cross-sectional design cannot confirm the apparent dissolution of a Latino nativity advantage in subsequent generations. It is possible that the relationship reflects a cohort effect rather than an effect of acculturation. If immigrant Latinos today are hardier than their counterparts of earlier generations, this would render as spurious the apparent attenuation of the nativity advantage. Alternatively, the host environment today may be more conducive to promoting Latino oral health related quality of life. Latinos now migrate into a much larger and growing Latino population in the host country with greater opportunity for social networks and expanding community services. Trend data over two decades 1979-81 to 1999-2001 supports this argument in showing that the Latino mortality advantage has increased (Singh \& Hiatt, 2006).

The decision to distinguish between Latinos solely on the basis of nativity status oversimplifies the heterogeneous nature of this population potentially masking important variation. Although 
the Latino population shares a Spanish language background, Latin America is a culturally, racially and ethnically diverse region comprising more than 20 countries throughout North, Central and South America and the Caribbean. Latino immigrants vary in socio-political history, reason for migration, socioeconomic attainment and opportunity for assimilation into mainstream society as well as in risk for disease. Whether the health advantage is generalizable to immigrants from all Latino countries cannot be determined in this analysis. Notwithstanding the crude dichotomy that tends to bias findings towards the null, this study revealed dramatic differences in oral health-related quality of life between immigrant Latinos and the non-Latino majority white population.

The Latino advantage in oral health related quality of life is limited to immigrant Latinos, and is thus a nativity advantage as opposed to an ethnicity one. This fact, coupled with its apparent diminution over time, implies a protective effect of Latin American culture rather than an attribute of the individuals themselves. Understanding this attribute lies at the heart of the nation's major public health goals to improve health related quality of life and eliminate health disparities (U.S. Department of Health and Human Services, November 2000).

There is a need for studies with sufficient large sample size to account for the full heterogeneity of the Latino population so that comparisons can be made of Latino immigrants from different countries. Future studies should also consider the role of length of residence in country of birth and in the United States, and how exposure to the two environments my contribute to the Latino advantage in oral health-related quality of life for immigrants and its loss in subsequent generations.

Public health inquiry will be further informed by determining whether Latino immigrants to other countries such as Canada, also an advantage in oral health-related quality of life, or whether the effect is evident only in the United States.

\section{Acknowledgments}

This work was supported by the National Institute of Dental and Craniofacial Research at the National Institutes of Health [grant number R21-DE018980].

\section{REFERENCES}

Abraido-Lanza AF, Dohrenwend BP, Ng-Mak DS, Turner JB. The Latino mortality paradox: a test of the "salmon bias" and healthy migrant hypotheses. Am J Public Health 1999;89(10):1543-1548. [PubMed: 10511837]

Alderete E, Vega WA, Kolody B, Aguilar-Gaxiola S. Lifetime prevalence of and risk factors for psychiatric disorders among Mexican migrant farmworkers in California. Am J Public Health 2000;90 (4):608-614. [PubMed: 10754977]

Alegria M, Canino G, Shrout PE, Woo M, Duan N, Vila D, et al. Prevalence of mental illness in immigrant and non-immigrant U.S. Latino groups. Am J Psychiatry 2008;165(3):359-369. [PubMed: 18245178]

Almeida J, Molnar BE, Kawachi I, Subramanian SV. Ethnicity and nativity status as determinants of perceived social support: testing the concept of familism. Soc Sci Med 2009;68(10):1852-1858. [PubMed: 19303184]

Arias E, Schauman WS, Eschbach K, Sorlie PD, Backlund E. The validity of race and Hispanic origin reporting on death certificates in the United States. Vital Health Stat 2008;2(148):1-23.

Borrell LN, Crawford ND. Social disparities in periodontitis among United States adults 1999-2004. Community Dent Oral Epidemiol 2008;36(5):383-391. [PubMed: 18924254]

Brennan DS, Spencer AJ. Life events and oral-health-related quality of life among young adults. Qual Life Res 2009;18(5):557-565. [PubMed: 19404772]

Coburn D. Beyond the income inequality hypothesis: class, neo-liberalism, and health inequalities. Soc Sci Med 2004;58(1):41-56. [PubMed: 14572920] 
Franzini L, Ribble JC, Keddie AM. Understanding the Hispanic paradox. Ethn Dis 2001;11(3):496-518. [PubMed: 11572416]

Hessol NA, Fuentes-Afflick E. The perinatal advantage of Mexican-origin Latina women. Ann Epidemiol 2000;10(8):516-523. [PubMed: 11118931]

Hummer RA, Powers DA, Pullum SG, Gossman GL, Frisbie WP. Paradox found (again): infant mortality among the Mexican-origin population in the United States. Demography 2007;44(3):441-457. [PubMed: 17913005]

Liao Y, Cooper RS, Cao G, Durazo-Arvizu R, Kaufman JS, Luke A, et al. Mortality patterns among adult Hispanics: findings from the NHIS, 1986 to 1990. Am J Public Health 1998;88(2):227-232. [PubMed: 9491012]

Locker D. Measuring oral health: a conceptual framework. Community Dent Health 1988;5(1):3-18. [PubMed: 3285972]

Lopez R, Baelum V. Spanish version of the Oral Health Impact Profile (OHIP-Sp). BMC Oral Health 2006;6:11. [PubMed: 16827940]

Markides KS, Eschbach K. Aging, migration, and mortality: current status of research on the Hispanic paradox. J Gerontol B Psychol Sci Soc Sci 2005;60:68-75. Spec No 2. [PubMed: 16251594]

Melchior M, Berkman LF, Niedhammer I, Chea M, Goldberg M. Social relations and self-reported health: a prospective analysis of the French Gazel cohort. Soc Sci Med 2003;56(8):1817-1830. [PubMed: 12639598]

Moorman JE, Rudd RA, Johnson CA, King M, Minor P, Bailey C, et al. National surveillance for asthma-United States, 1980-2004. MMWR Surveill Summ 2007;56(8):1-54. [PubMed: 17947969]

Moreno-Brid JC, Pardinas Carpizo JE, Ros Bosch J. Economic development and social policies in Mexico. Economy and Society 2009;38:154-176.

OECD. Society at a Glance 2009 - OECD Social Indicators. 2009

Patrick DL, Lee RS, Nucci M, Grembowski D, Jolles CZ, Milgrom P. Reducing oral health disparities: a focus on social and cultural determinants. BMC Oral Health 2006;6:S4. [PubMed: 16934121]

Pena JB, Wyman PA, Brown CH, Matthieu MM, Olivares TE, Hartel D, et al. Immigration generation status and its association with suicide attempts, substance use, and depressive symptoms among latino adolescents in the USA. Prev Sci 2008;9(4):299-310. [PubMed: 18855139]

Rubalcava LN, Teruel GM, Thomas D, Goldman N. The healthy migrant effect: new findings from the Mexican Family Life Survey. Am J Public Health 2008;98(1):78-84. [PubMed: 18048791]

Sabbah W, Tsakos G, Chandola T, Sheiham A, Watt RG. Social gradients in oral and general health. J Dent Res 2007;86(10):992-996. [PubMed: 17890677]

Sanders AE, Slade GD, John MT, Steele JG, Suominen-Taipale AL, Lahti S, et al. A cross-national comparison of income gradients in oral health quality-of-life in four welfare states: application of the Korpi and Palme typology. J Epidemiol Community Health. 2009a

Sanders AE, Slade GD, John MT, Steele JG, Suominen-Taipale AL, Lahti S, et al. A cross-national comparison of income gradients in oral health quality of life in four welfare states: application of the Korpi and Palme typology. J Epidemiol Community Health 2009b;63(7):569-574. [PubMed: 19351621]

Sanders AE, Slade GD, Lim S, Reisine ST. Impact of oral disease on quality of life in the US and Australian populations. Community Dent Oral Epidemiol 2009;37(2):171-181. [PubMed: 19175659]

Segura-Ubiergo, A. The Political Economy of the Welfare State in Latin America: Globalization, Democracy, and Development. Cambridge; New York: Cambridge University Press; 2007.

Singh GK, Hiatt RA. Trends and disparities in socioeconomic and behavioural characteristics, life expectancy, and cause-specific mortality of native-born and foreign-born populations in the United States, 1979-2003. Int J Epidemiol 2006;35(4):903-919. [PubMed: 16709619]

Slade GD. Derivation and validation of a short-form oral health impact profile. Community Dent Oral Epidemiol 1997;25(4):284-290. [PubMed: 9332805]

Slade GD, Spencer AJ. Development and evaluation of the Oral Health Impact Profile. Community Dent Health 1994;11(1):3-11. [PubMed: 8193981] 
Sorlie PD, Backlund E, Johnson NJ, Rogot E. Mortality by Hispanic status in the United States. JAMA 1993;270(20):2464-2468. [PubMed: 8031341]

Steele JG, Sanders AE, Slade GD, Allen PF, Lahti S, Nuttall N, et al. How do age and tooth loss affect oral health impacts and quality of life? A study comparing two national samples. Community Dent Oral Epidemiol 2004;32(2):107-114. [PubMed: 15061859]

Subramanian SV, Jun HJ, Kawachi I, Wright RJ. Contribution of race/ethnicity and country of origin to variations in lifetime reported asthma: evidence for a nativity advantage. Am J Public Health 2009;99 (4):690-697. [PubMed: 19218175]

Thomas AJ, Eberly LE, Neaton JD, Smith GD. Latino risk-adjusted mortality in the men screened for the Multiple Risk Factor Intervention Trial. Am J Epidemiol 2005;162(6):569-578. [PubMed: 16076831]

Turra CM, Elo IT. The Impact of Salmon Bias on the Hispanic Mortality Advantage: New Evidence from Social Security Data. Popul Res Policy Rev 2008;27(5):515-530. [PubMed: 19122882]

Turra CM, Goldman N. Socioeconomic differences in mortality among U.S. adults: insights into the Hispanic paradox. J Gerontol B Psychol Sci Soc Sci 2007;62(3):S184-S192. [PubMed: 17507594]

U.S. Department of Health and Human Services. Healthy People 2010. 2nd ed. With Understanding and Improving Health and Objectives for Improving Health. Vol. 2 vols.. Washington, DC: U.S. Government Printing Office; 2000 Nov.

World Health Organization. International Classification of Impairments Disabilities and Handicaps: a manual of classification. Geneva: 1980. p. 23-42. 


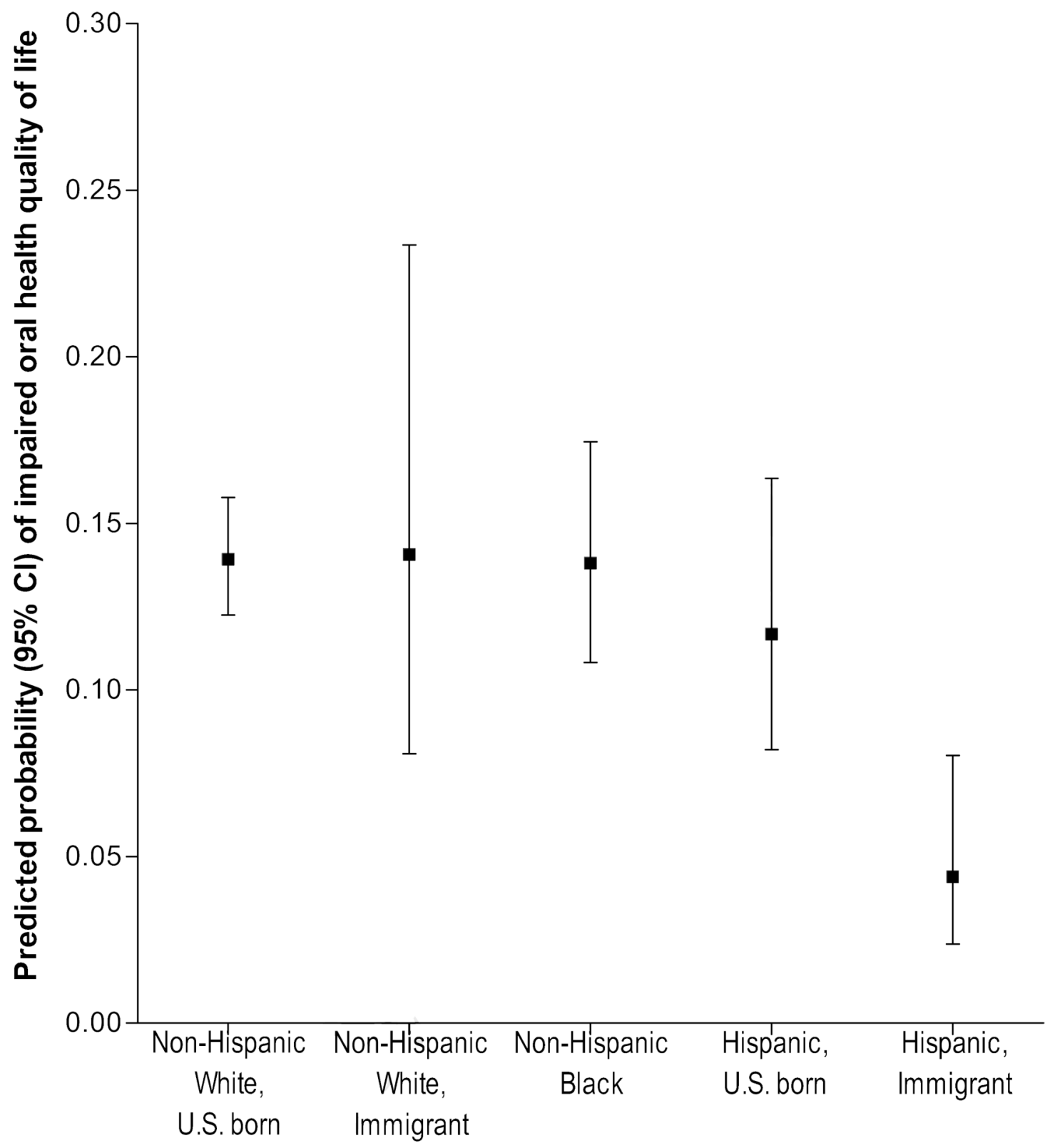

\section{Race/ethnicity and nativity}

Figure 1.

Adjusted prevalence and $95 \%$ confidence intervals of impaired oral health-related quality of life according to racial/ethnic and nativity status, National Health and Nutrition Examination Survey, 2003-2004, United States. 


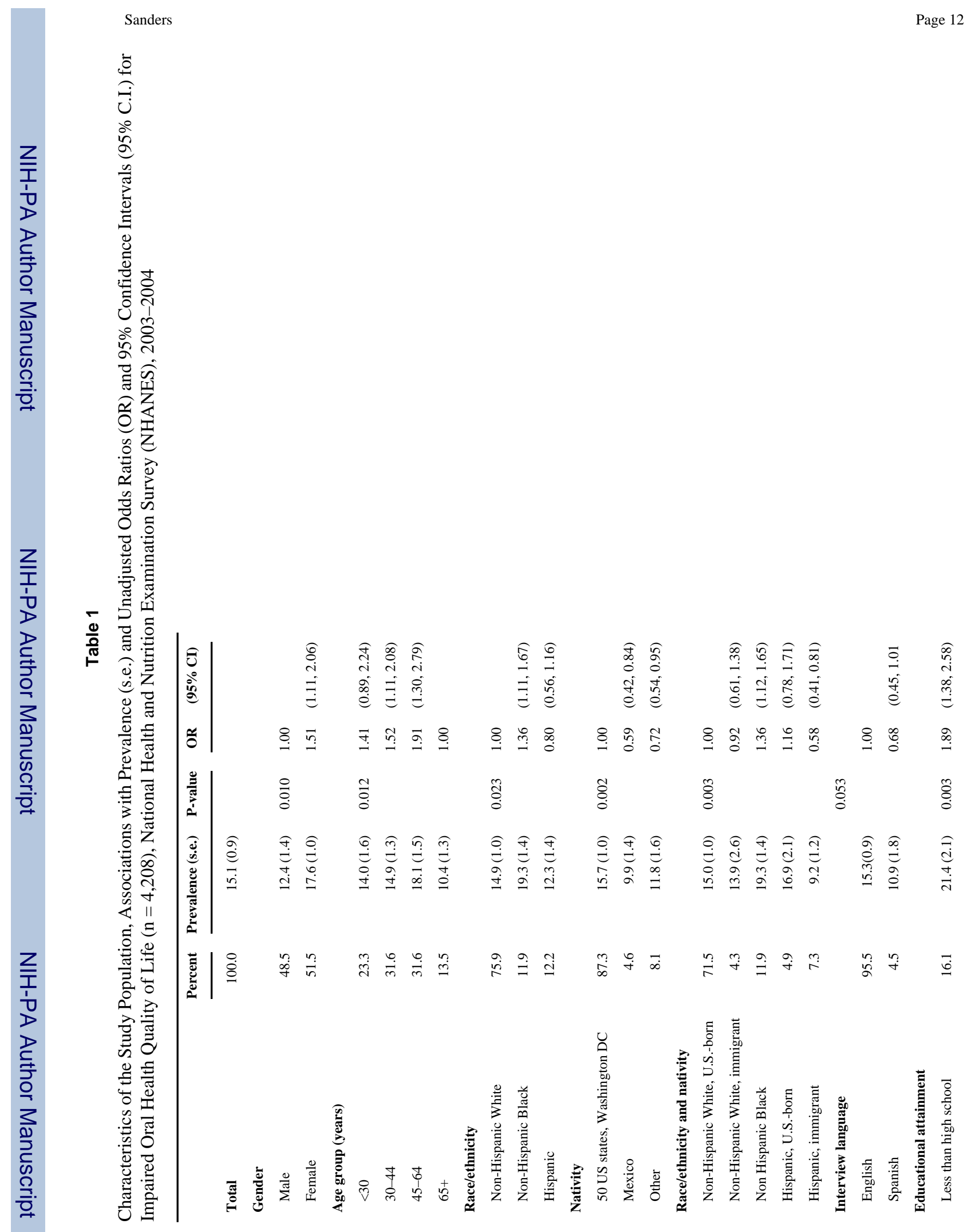

Soc Sci Med. Author manuscript; available in PMC 2011 July 1. 


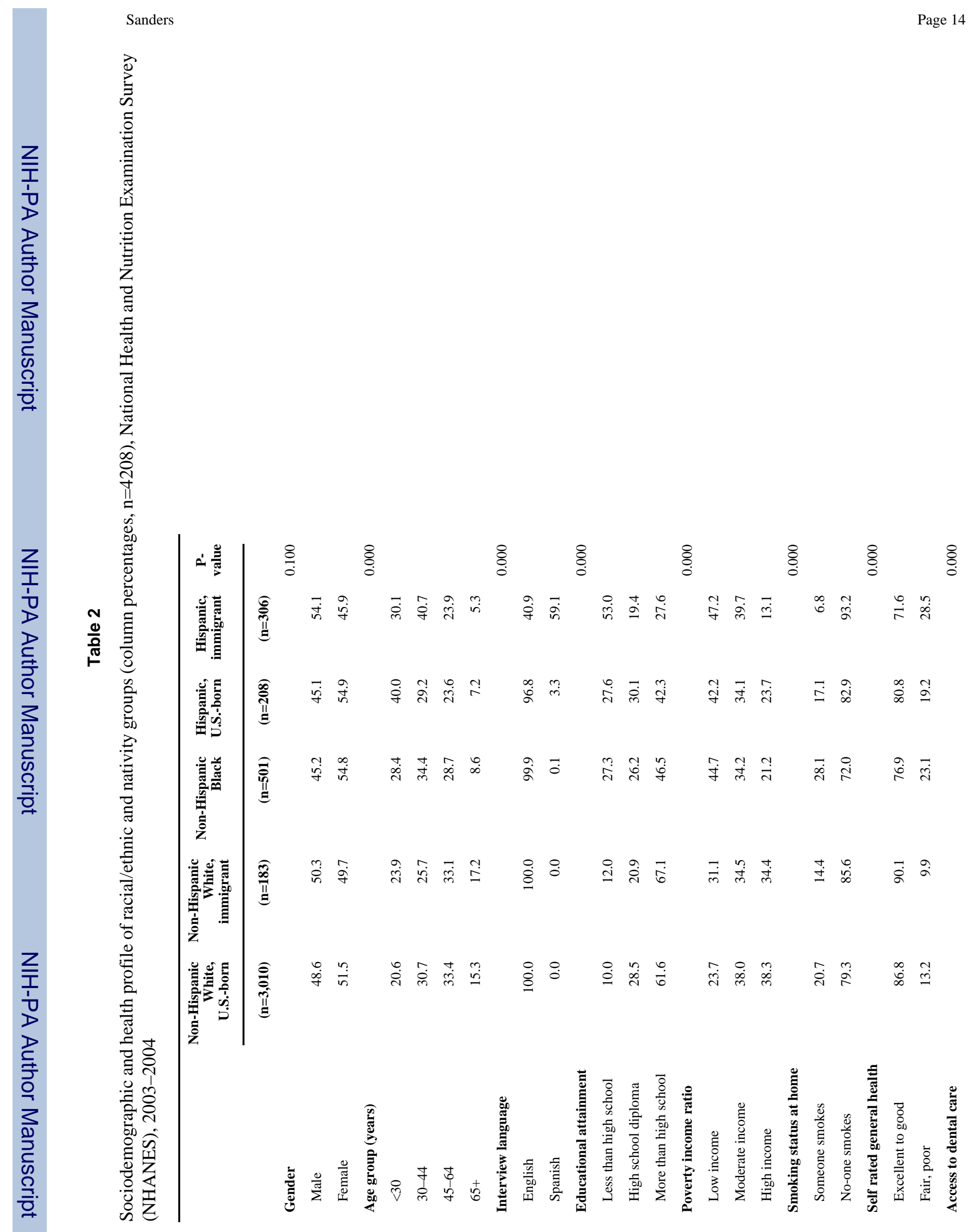

Soc Sci Med. Author manuscript; available in PMC 2011 July 1. 


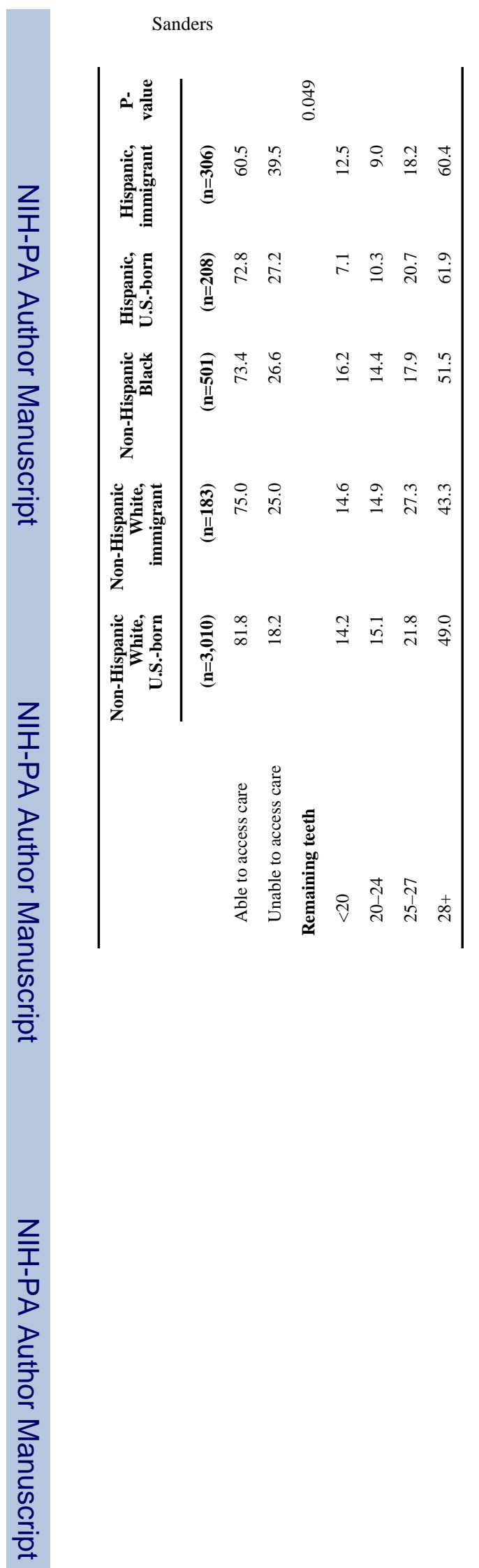

Soc Sci Med. Author manuscript; available in PMC 2011 July 1. 


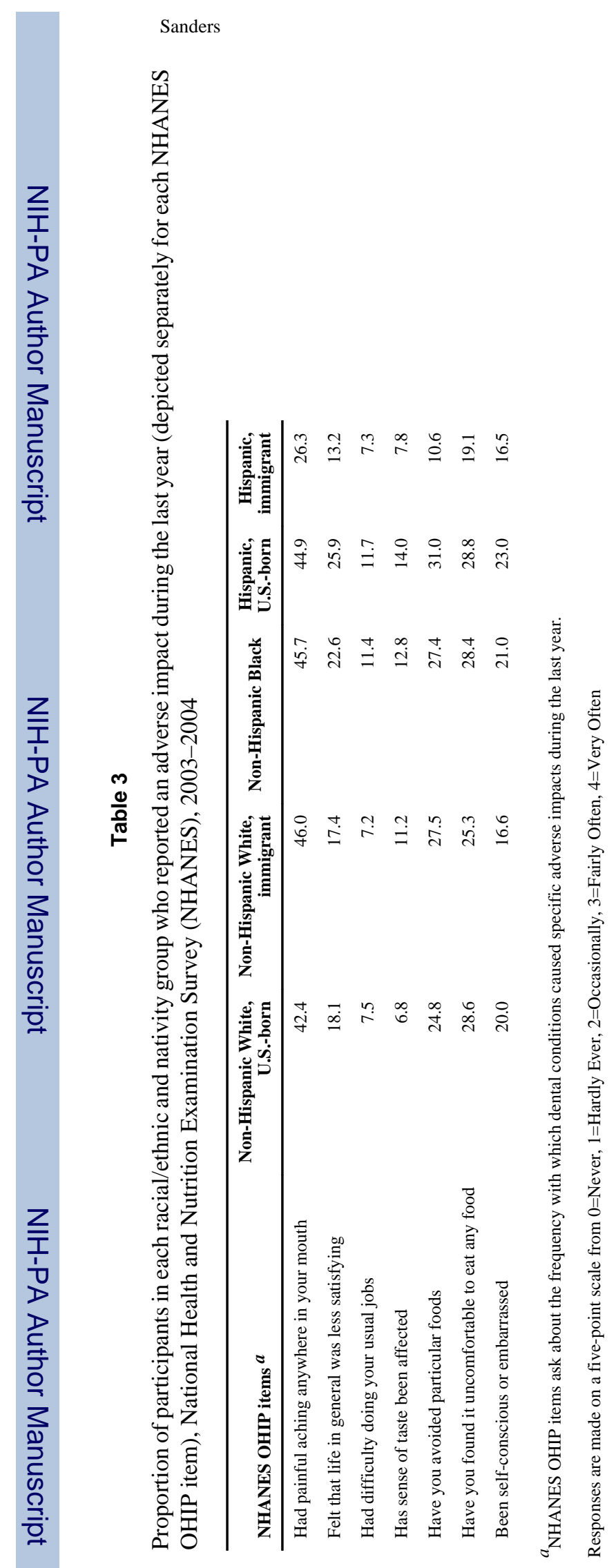

Soc Sci Med. Author manuscript; available in PMC 2011 July 1. 


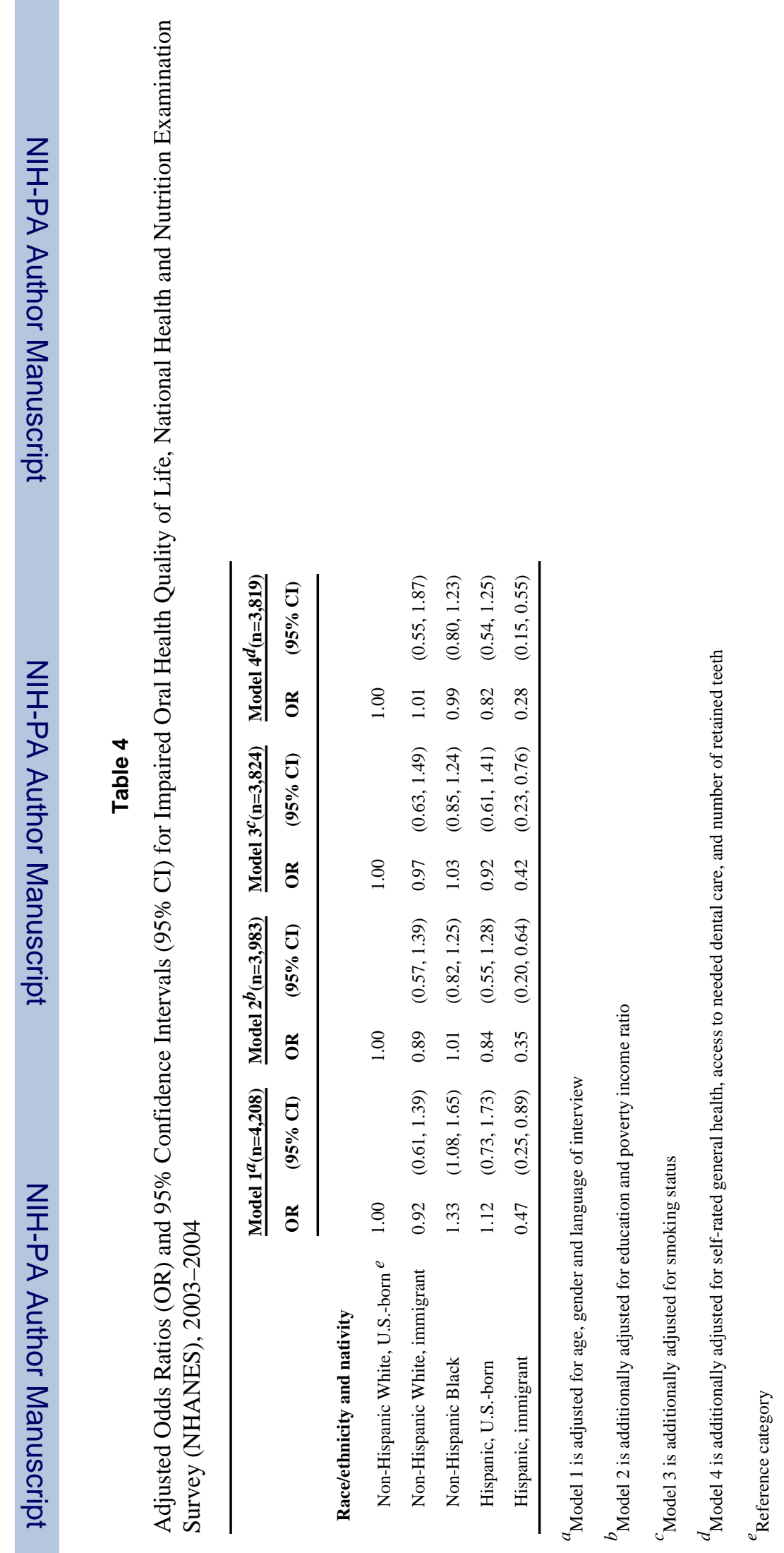

